\title{
Controle Ótimo de Pragas em Vários Estágios do seu Desenvolvimento
}

\author{
L.C. SAKR ${ }^{1}$, L. LUETKEMEYER, M. RAFIKOV ${ }^{2}$, Programa de Pós-Graduação \\ em Modelagem Matemática, UNIJUÍ, Cx.P. 560, 98700-000 Ijuí, RS, Brasil.
}

Resumo. Nos últimos anos uma das mais relevantes conquistas do controle de pragas foi a elaboração e implantação da tecnologia de aplicação do Baculovirus nas lavouras de soja, que mostra a sua eficácia contra a lagarta da soja Anticarsia gemmatalis. No entanto, uma das limitações principais do uso do Baculovirus é o clima. A aplicação de Baculovirus tem maior eficácia com o tempo chuvoso quando a doença se espalha rapidamente na lavoura e permanece por um longo tempo. Quando o tempo é seco, como aconteceu, por exemplo, na safra de 1999/2000 no Rio Grande do Sul, a eficácia do Baculovirus e outras doenças de pragas da soja diminuem muito. Existe também a ameaça permanente de que a aplicação do Baculovirus e outros patógenos, durante longo tempo, inicie uma seleção nas gerações de pragas mais resistentes contra as doenças. Essas razões levam os pesquisadores a pensar em outras alternativas para o controle biológico de pragas. Um tipo de controle biológico, em muitos casos bem sucedido, é o lançamento de parasitóides.

Neste trabalho foi considerado um modelo matemático formado por cinco equações diferenciais que descrevem os estágios de desenvolvimento das pragas e a dinâmica das interações entre elas e seus parasitóides. Foi formulado um problema de otimização de aplicações de controle, que minimiza a população de pragas e os custos das aplicações e maximiza a população de inimigos naturais. O problema foi resolvido através do Princípio do Máximo de Pontryagin [1].

\section{Introdução}

O controle das pragas que atacam as lavouras sempre foi um grande motivo de preocupação para as pessoas que trabalham com a agricultura. O controle químico através da aplicação de inseticidas, que inicialmente pareceu ter resolvido o problema, já não é mais uma unanimidade. Além de ser um método dispendioso tem efeitos colaterais por vezes mais maléficos que as pragas combatidas. Quando utilizado de forma única, o controle químico, pode deixar resíduos nos alimentos, comprometer mananciais, prejudicar a saúde e em casos mais graves causar o óbito dos operadores, além de provocar o ressurgimento de pragas mais resistentes e desequilibrar o meio ambiente. O conhecimento destas desvantagens fez crescer o interesse

\footnotetext{
${ }^{1}$ sluiz@unc-cni.rct-sc.br

${ }^{2}$ rafikov@main.unijui.tche.br
} 
por controles alternativos, especialmente o controle biológico, o qual faz uso de inimigos naturais para conter populações tanto animais quanto vegetais prejudiciais ao homem.

O controle biológico pode atuar como um auxiliar no combate as pragas ou até mesmo como a única forma de combate. A decisão depende, dentre outras razões, da quantidade de pragas existentes, da forma como atua o inimigo natural, das condições climáticas e do tempo que se dispõe para o combate.

Uma das formas de controle biológico utilizada pelos produtores de soja é a aplicação do Baculovirus. Dependendo das condições em que esta tecnologia é utilizada seus efeitos são de tal forma favoráveis que levou alguns agricultores a pensar que o controle de pragas, principalmente a lagarta da soja Anticarsia gemmatalis, estava satisfatória e definitivamente resolvido. No entanto, o clima, fator importante para a aplicação desta técnica não foi favorável nesta safra 1999/2000. A ausência de chuvas comprometeu a eficácia da atuação do Baculovirus. Sendo uma técnica de ataque as pragas com a finalidade de adoecê-las, o Baculovirus resiste mais tempo e dissemina-se mais rapidamente em condições chuvosas. Também não se pode esquecer que, mesmo quando eficaz, o Baculovirus, se utilizado sistematicamente pode promover a seleção natural de pragas resistentes à sua atuação inviabilizando o processo e dificultando ainda mais o combate.

Outra alternativa de controle biológico, da qual se ocupará este trabalho, é a utilização de parasitóides no combate às pragas da soja. Os parasitóides são insetos que utilizam a lagarta como hospedeira de seus ovos, causando a sua morte. Mesmo sendo uma forma de combate satisfatória, também tem suas dificuldades. Uma das principais é a falta de informações que o agricultor dispõe sobre o momento adequado e sobre a quantidade adequada de parasitóides que devem ser lançados para o combate às pragas. Opta geralmente pelo chamado método inundativo através do qual simplesmente inunda a plantação com parasitóides, independente da quantidade destes ser superior à necessária. No entanto: considerando que nem sempre o extermínio das pragas é desejável uma vez que é preciso manter o equilíbrio biológico, para que os parasitóides não precisem abandonar o local pela ausência de pragas que hospedem seus ovos; considerando que os parasitóides muitas vezes não são nativos da região, nestes casos precisando ser trazidos até de outros países, origem das pragas; considerando que em certos casos os parasitóides necessitam ser desenvolvidos em laboratórios, torna-se importante a sua utilização racional, no momento apropriado e sem exageros.

Para obter essas informações, que possibilitarão ao agricultor empregar com segurança, eficácia e economia o controle biológico através da utilização de parasitóides, este trabalho considera um modelo matemático formado por cinco equações diferenciais. Estas equações, compõe o sistema (1.1) e descrevem a dinâmica das interações entre pragas da soja e seus parasitóides, admitindo estágios de desenvolvimento das pragas, competição entre espécies de um estágio e considerando também aplicações de controle. 


$$
\begin{aligned}
\frac{d B}{d t}= & k_{p}\left(P-U_{4}\right)-\gamma_{b}\left(B-U_{1}\right)^{2}-\mu_{b}\left(B-U_{1}\right)-k_{1} U_{1} \\
\frac{d O}{d t}= & q\left(B-U_{1}\right)-\gamma_{o}\left(O-U_{2}\right)^{2}-\mu_{o}\left(O-U_{2}\right)-k_{o}\left(O-U_{2}\right)-k_{2} U_{2} \\
\frac{d X}{d t}= & k_{o}\left(O-U_{2}\right)-\gamma_{x}\left(X-U_{3}\right)^{2}-\mu_{x}\left(X-U_{3}\right)-\alpha\left(X-U_{3}\right)(Y+V) \\
& \quad-k_{x}\left(X-U_{3}\right)-k_{3} U_{3} \\
\frac{d P}{d t}= & k_{x}\left(X-U_{3}\right)-\gamma_{p}\left(P-U_{4}\right)^{2}-\mu_{p}\left(P-U_{4}\right)-k_{p}\left(P-U_{4}\right)-k_{4} U_{4} \\
\frac{d Y}{d t}= & \beta\left(X-U_{3}\right)(Y+V)-\mu_{y}(Y+V)+k_{5} V
\end{aligned}
$$

onde:

$B, O, X, P$ e $Y=$ densidade por $m^{2}$ no instante $t$, respectivamente, de mariposas, de ovos, de lagartas, de pupas e de parasitóides;

$k_{p}=$ coeficiente que caracteriza que parte das pupas tornam-se mariposas, no instante $t$;

$k_{o}=$ coeficiente que caracteriza que parte dos ovos tornaram-se larvas, no instante $t$;

$k_{x}=$ coeficiente que caracteriza que parte das lagartas tornaram-se pupas, no instante $t$;

$\gamma_{b}, \gamma_{o}, \gamma_{x}, \gamma_{p}=$ coeficiente de eliminação, respectivamente, das mariposas, dos ovos, das lagartas e das pupas pela competição entre espécies de um estágio;

$\mu_{b}, \mu_{o}, \mu_{x}, \mu_{p}, \mu_{y}=$ coeficiente de mortalidade, respectivamente, das mariposas, dos ovos, das lagartas, das pupas e dos parasitóides;

$q=$ coeficiente que caracteriza o fluxo de oviposição por par de mariposas;

$\alpha=$ coeficiente de eliminação das lagartas pela parasitação;

$\beta=$ coeficiente de natalidade dos parasitóides pela parasitação;

$U_{1}(t), U_{2}(t), U_{3}(t)$ e $U_{4}(t)$ correspondem, respectivamente, ao número de mariposas, ovos, lagartas e pupas retiradas do sistema no instante $t$;

$V(t)$ corresponde ao número de parasitóides introduzidos no sistema no instante $t$

$k_{1}, k_{2}, k_{3}, k_{4}$ e $k_{5}$ são constantes positivas.

Com as condições iniciais:

$$
B(0)=B_{0} ; O(0)=O_{0} ; X(0)=X_{0} ; P(0)=P_{0} ; Y(0)=Y_{0}
$$

e as limitações:

$$
0 \leq U_{1} \leq B ; \quad 0 \leq U_{2} \leq O ; \quad 0 \leq U_{3} \leq X ; \quad 0 \leq U_{4} \leq P ; \quad 0 \leq V .
$$

Supondo que se deseja manter o nível de pragas abaixo do nível de danos econômicos e ter um baixo custo no uso de aplicações de controles, é necessária a utilização do critério de otimização:

$$
\begin{gathered}
I=c_{1}\left[B(T)+k_{1} \int_{t_{0}}^{T} U_{1}(t) d t\right]+c_{2}\left[O(T)+k_{2} \int_{t_{0}}^{T} U_{2}(t) d t\right]+c_{3}[X(T)+ \\
\left.k_{3} \int_{t_{0}}^{T} U_{3}(t) d t\right]+c_{4}\left[P(T)+k_{4} \int_{t_{0}}^{T} U_{4}(t) d t\right]+c_{5}\left[k_{5} \int_{t_{0}}^{T} V(t) d t-Y(T)\right]
\end{gathered}
$$


onde:

$c_{1}, c_{2}, c_{3}, c_{4}$ e $c_{5}$ são constantes positivas que caracterizam o peso de cada tipo de controle;

$t_{0}$ e $T$ são os momentos inicial e final da aplicação do controle, respectivamente.

Minimizar o critério (1.4) é minimizar os valores das funções de controle e das populações de pragas durante o período da aplicação e maximizar a população de inimigos naturais.

Logo, o problema do controle ótimo de pragas pode ser formulado como:

"Encontrar as funções de controle $U_{i}(t), i=1,2,3$ e 4 e $V(t)$ que minimizam o funcional (1.4) e satisfazem o sistema (1.1) e as condições (1.2) e (1.3)".

\section{Resolução do Problema}

Para maior comodidade na solução é conveniente introduzir uma nova função :

$$
\begin{aligned}
& W(t)=c_{1}\left[B(t)+k_{1} \int_{t_{0}}^{t} U_{1}(t) d t\right]+c_{2}\left[O(t)+k_{2} \int_{t_{0}}^{t} U_{2}(t) d t\right]+c_{3}[X(t) \\
& \left.+k_{3} \int_{t_{0}}^{t} U_{3}(t) d t\right]+c_{4}\left[P(t)+k_{4} \int_{t_{0}}^{t} U_{4}(t) d t\right]+c_{5}\left[k_{5} \int_{t_{0}}^{t} V(t) d t-Y(t)\right],
\end{aligned}
$$

cuja derivada com o emprego do sistema (1.1), será:

$$
\begin{aligned}
& \frac{d W(t)}{d t}=c_{1}\left[k_{p}\left(P-U_{4}\right)-\gamma_{b}\left(B-U_{1}\right)^{2}-\mu_{b}\left(B-U_{1}\right)-k_{1} U_{1}+k_{1} U_{1}\right] \\
& +c_{2}\left[q\left(B-U_{1}\right)-\gamma_{o}\left(O-U_{2}\right)^{2}-\mu_{o}\left(O-U_{2}\right)-k_{o}\left(O-U_{2}\right)-k_{2} U_{2}+k_{2} U_{2}\right] \\
& +c_{3}\left[\begin{array}{c}
k_{o}\left(O-U_{2}\right)-\gamma_{x}\left(X-U_{3}\right)^{2}-\mu_{x}\left(X-U_{3}\right) \\
-\alpha\left(X-U_{3}\right)(Y+V)-k_{x}\left(X-U_{3}\right)-k_{3} U_{3}+k_{3} U_{3}
\end{array}\right] \\
& +c_{4}\left[k_{x}\left(X-U_{3}\right)-\gamma_{p}\left(P-U_{4}\right)^{2}-\mu_{p}\left(P-U_{4}\right)-k_{p}\left(P-U_{4}\right)-k_{4} U_{4}+k_{4} U_{4}\right] \\
& +c_{5}\left[k_{5} V-\beta\left(X-U_{3}\right)(Y+V)+\mu_{y}(Y+V)-k_{5} V\right] .
\end{aligned}
$$

Denota-se:

$$
\begin{aligned}
& \xi_{1}=B-U_{1}(t) ; \quad \xi_{2}=O-U_{2}(t) ; \quad \xi_{3}=X-U_{3}(t) \\
& \xi_{4}=P-U_{4}(t) ; \quad \eta=Y+V(t)
\end{aligned}
$$

e emprega-se a função de Hamilton:

$$
H=\lambda_{0} \frac{d W}{d t}+\lambda_{1} \frac{d B}{d t}+\lambda_{2} \frac{d O}{d t}+\lambda_{3} \frac{d X}{d t}+\lambda_{4} \frac{d P}{d t}+\lambda_{5} \frac{d Y}{d t},
$$

onde:

$\lambda_{0}, \lambda_{1}, \lambda_{2}, \lambda_{3}, \lambda_{4}$ e $\lambda_{5}$ são variáveis conjugadas determinadas pelas equações: 


$$
\begin{array}{rlrl}
\frac{d \lambda_{0}}{d t} & =-\frac{\partial H}{\partial W}=0 ; & \frac{d \lambda_{1}}{d t}=-\frac{\partial H}{\partial B} ; & \frac{d \lambda_{2}}{d t}=-\frac{\partial H}{\partial O} ; \\
\frac{d \lambda_{3}}{d t}=-\frac{\partial H}{\partial X} ; & \frac{d \lambda_{4}}{d t}=-\frac{\partial H}{\partial P} ; & \frac{d \lambda_{5}}{d t}=-\frac{\partial H}{\partial Y} ;
\end{array}
$$

com as condições finais:

$$
\lambda_{0}(T)=-1 ; \quad \lambda_{i}(T)=0, \quad i=1,2,3,4,5 .
$$

Conforme o Princípio do Máximo de Pontryagin, as funções de controle ótimo, maximizam a função e as condições necessárias para o máximo da função são:

$$
\begin{array}{ll}
\frac{\partial H}{\partial U_{1}}=-\frac{\partial H}{\partial \xi_{1}}-k_{1} \lambda_{1}=0 ; & \frac{\partial H}{\partial U_{2}}=-\frac{\partial H}{\partial \xi_{2}}-k_{2} \lambda_{2}=0 ; \quad \frac{\partial H}{\partial U_{3}}=-\frac{\partial H}{\partial \xi_{3}}-k_{3} \lambda_{3}=0 ; \\
\frac{\partial H}{\partial U_{4}}=-\frac{\partial H}{\partial \xi_{4}}-k_{4} \lambda_{4}=0 ; & \frac{\partial H}{\partial V}=-\frac{\partial H}{\partial \xi_{5}}-k_{5} \lambda_{5}=0 ;
\end{array}
$$

Da primeira equação do sistema (2.5), tem-se: $\lambda_{0}=$ constante, que associando à condição final (2.6), leva a:

$$
\lambda_{0}=-1
$$

De (2.5), (2.3) e (2.7), tem-se:

$$
\begin{aligned}
& \frac{d \lambda_{1}}{d t}=-\frac{\partial H}{\partial B}=-\frac{\partial H}{\partial \xi_{1}} \cdot \frac{\partial \xi_{1}}{\partial B}=-\left(-k_{1} \lambda_{1}\right) \cdot(1) \quad \Longrightarrow \quad \frac{d \lambda_{1}}{d t}=k_{1} \lambda_{1} \\
& \frac{d \lambda_{2}}{d t}=-\frac{\partial H}{\partial O}=-\frac{\partial H}{\partial \xi_{2}} \cdot \frac{\partial \xi_{2}}{\partial O}=-\left(-k_{2} \lambda_{2}\right) \cdot(1) \quad \Longrightarrow \quad \frac{d \lambda_{2}}{d t}=k_{2} \lambda_{2}, \\
& \frac{d \lambda_{3}}{d t}=-\frac{\partial H}{\partial X}=-\frac{\partial H}{\partial \xi_{3}} \cdot \frac{\partial \xi_{3}}{\partial X}=-\left(-k_{3} \lambda_{3}\right) \cdot(1) \quad \Longrightarrow \quad \frac{d \lambda_{3}}{d t}=k_{3} \lambda_{3}, \\
& \frac{d \lambda_{4}}{d t}=-\frac{\partial H}{\partial P}=-\frac{\partial H}{\partial \xi_{4}} \cdot \frac{\partial \xi_{4}}{\partial B}=-\left(-k_{4} \lambda_{4}\right) \cdot(1) \quad \Longrightarrow \quad \frac{d \lambda_{4}}{d t}=k_{4} \lambda_{4}, \\
& \frac{d \lambda_{5}}{d t}=-\frac{\partial H}{\partial Y}=-\frac{\partial H}{\partial \eta} \cdot \frac{\partial \eta}{\partial Y}=-\left(-k_{5} \lambda_{5}\right) \cdot(1) \quad \Longrightarrow \quad \frac{d \lambda_{5}}{d t}=k_{5} \lambda_{5}
\end{aligned}
$$

Considerando as soluções gerais de (2.9), (2.10), (2.11), (2.12) e (2.13) e associando à condição final (2.6) chega-se a:

$$
\lambda_{1}(t)=\lambda_{2}(t)=\lambda_{3}(t)=\lambda_{4}(t)=\lambda_{5}(t) \equiv 0 .
$$

Aplicando (2.8) e (2.14) em (2.7), obtém-se: 


$$
\begin{aligned}
& \xi_{1}=\frac{c_{2} q-c_{1} \mu_{b}}{2 c_{1} \gamma_{b}}, \quad \xi_{2}=\frac{c_{3} k_{0}-c_{2} k_{0}-c_{2} \mu_{0}}{2 c_{2} \gamma_{0}}, \\
& \xi_{3}=\frac{c_{5} \mu_{y}}{c_{3} \alpha+c_{5} \beta}, \quad \xi_{4}=\frac{c_{1} k_{p}-c_{4} \mu_{p}-c_{4} k_{p}}{2 c_{4} \gamma_{p}} \\
& \eta=\frac{c_{4} k_{x}-c_{3} k_{x}-c_{3} \mu_{x}}{c_{3}+c_{5} \beta}-\frac{2 c_{3} c_{5} \gamma_{y}}{\left(c_{3} \alpha+c_{5} \beta\right)^{2}} .
\end{aligned}
$$

Determinando os valores de $\xi_{1}, \xi_{2}, \xi_{3}, \xi_{4}$ e $\eta$ através de (2.16) e em razão de (1.3) e (2.3) encontram-se as funções ótimas de controle:

$$
\begin{gathered}
U_{1}(t)=\left\{\begin{array}{lll}
B-\xi_{1} & \text { quando } & B>\xi_{1} \\
0 & \text { quando } & B \leq \xi_{1}
\end{array},\right. \\
U_{2}(t)=\left\{\begin{array}{lll}
O-\xi_{2} & \text { quando } & O>\xi_{2} \\
0 & \text { quando } & O \leq \xi_{2}
\end{array},\right. \\
U_{3}(t)=\left\{\begin{array}{lll}
X-\xi_{3} & \text { quando } & X>\xi_{3} \\
0 & \text { quando } & X \leq \xi_{3}
\end{array},\right. \\
U_{4}(t)=\left\{\begin{array}{lll}
P-\xi_{4} & \text { quando } & P>\xi_{4} \\
0 & \text { quando } & P \leq \xi_{4}
\end{array},\right. \\
V(t)=\left\{\begin{array}{lll}
\eta-Y & \text { quando } & Y<\eta \\
0 & \text { quando } & Y \geq \eta
\end{array},\right.
\end{gathered}
$$

\section{Determinação das Constantes: $c_{1}, c_{2}, c_{3}, c_{4}$ e $c_{5}$}

Como $\xi_{1}, \xi_{2}, \xi_{3}, \xi_{4}$ e $\eta$ representam, respectivamente, quantidades de mariposas, ovos, lagartas, pupas e parasitóides, o que os torna não negativos, tem-se de (2.16) que:

$$
\begin{aligned}
& \frac{c_{2} q-c_{1} \mu_{b}}{2 c_{1} \gamma_{b}} \geq 0, \quad \frac{c_{5} k_{0}-c_{2} k_{0}-c_{2} \mu_{0}}{2 c_{2} \gamma_{0}} \geq 0, \\
& \frac{c_{5} \mu_{y}}{c_{3} \alpha+c_{5} \beta} \geq 0, \quad \frac{c_{1} k_{p}-c_{4} \mu_{p}-c_{4} k_{p}}{2 c_{4} \gamma_{p}} \geq 0, \\
& \frac{c_{4} k_{x}-c_{3} k_{x}-c_{3} \mu_{x}}{c_{3} \alpha+c_{5} \beta}-\frac{2 c_{3} c_{5} \gamma_{x} \mu_{y}}{\left(c_{3} \alpha+c_{5} \beta\right)^{2}} \geq 0 .
\end{aligned}
$$

Uma vez que todas as constantes e todos os coeficientes presentes nas desigualdades (3.2) são positivos e que nas duas primeiras desigualdades temos como comum a constante $c_{2}$.

Obtém-se da primeira desigualdade que

$$
\frac{c_{1}}{c_{2}} \leq \frac{q}{\mu_{b}} .
$$


Denotando

$$
\overline{c_{1}}=\frac{c_{1}}{c_{2}}
$$

tem-se

$$
\overline{c_{1}} \leq \frac{q}{\mu_{b}} .
$$

Obtém-se da segunda desigualdade que

$$
\frac{c_{3}}{c_{2}} \geq \frac{k_{0}+\mu_{0}}{k_{0}} .
$$

Denotando

$$
\overline{c_{3}}=\frac{c_{3}}{c_{2}}
$$

tem-se

$$
\overline{c_{3}} \geq \frac{k_{0}+\mu_{0}}{k_{0}} .
$$

Obtém-se da quarta desigualdade que

$$
\frac{c_{4}}{c_{1}} \leq \frac{k_{p}}{k_{p}+\mu_{p}}
$$

Considerando (3.3) e denotando

$$
\overline{c_{4}}=\frac{c_{4}}{c_{2}}
$$

tem-se

$$
\overline{c_{4}} \leq \overline{c_{1}} \cdot \frac{k_{p}}{k_{p}+\mu_{p}} .
$$

Obtém-se da quinta desigualdade que

$$
c_{5} \geq \frac{c_{3}^{2} \alpha k_{x}+c_{3}^{2} \alpha \mu_{x}-c_{3} c_{4} \alpha k_{x}}{c_{4} \beta k_{x}-c_{3} \beta k_{x}-c_{3} \beta \mu_{x}-2 c_{3} \gamma_{x} \mu_{y}} .
$$

Considerando (3.6) e (3.9) e denotando

$$
\overline{c_{5}}=\frac{c_{5}}{c_{2}}
$$

tem-se

$$
c_{5} \geq \frac{{\overline{c_{3}}}^{2} \alpha\left(k_{x}+\mu_{x}\right)-\overline{c_{3} c_{4}} \alpha k_{x}}{\overline{c_{4}} \beta k_{x}-\overline{c_{3}}\left(\beta k_{x}+\beta \mu_{x}+2 \gamma_{x} \mu_{y}\right)}
$$

\section{Aplicações}

Utilizando os dados abaixo referentes a lagarta Pseudoplusia includens e o parasitóide Copidosoma truncatellum, seguem duas simulações numéricas. 


\begin{tabular}{|l|l|l|l|l|}
\hline & $k_{o}=0,462$ & $k_{x}=0,1066$ & $k_{p}=0,198$ & \\
\hline$\gamma_{b}=0,0001$ & $\gamma_{o}=0,0001$ & $\gamma_{x}=0,0001$ & $\gamma_{p}=0,0001$ & \\
\hline$\mu_{b}=0,1155$ & $\mu_{o}=0,4$ & $\mu_{x}=0,3$ & $\mu_{p}=0,2$ & $\mu_{y}=0,099$ \\
\hline & $q=2,2$ & $\alpha=0,001414$ & & $\beta=0,001414$ \\
\hline
\end{tabular}

Através de (3.4), (3.7), (3.10), (3.13), obtém-se para as constantes positivas $\overline{c_{1}}, \overline{c_{2}}, \overline{c_{3}}, \overline{c_{4}}$ e $\overline{c_{5}}$ :

$0 \leq \overline{c_{1}} \leq 19,05 ; \overline{c_{3}} \geq 1,87 ; 0 \leq \overline{c_{4}} \leq 0,4975 \overline{c_{1}} ; \overline{c_{5}} \geq \frac{0,000575{\overline{c_{3}}}^{2}-0,00015 \overline{c_{3} c_{4}}}{0,00015 \overline{c_{4}}-0,000595 \overline{c_{3}}}$.

$1^{a}$ SIMULAÇÃO: com aplicação de controle

Considerando valores para as constantes positivas que estabilizam a quantidade de lagartas em 40 por $\mathrm{m}^{2}$, quantidade considerada pela EMBRAPA - Empresa Brasileira de Pesquisa Agropecuária como aceitável (40 lagartas pequenas).

Atribuindo $\overline{c_{1}}=16,5, \overline{c_{3}}=2$, obtém-se $\overline{c_{4}}=8, \overline{c_{5}}=2$ e atribuindo para a constante $c_{2}$, anteriormente utilizada como referência o valor $c_{2}=2$ através de (3.3), (3.6), (3.11) e (3.12), têm-se: $c_{1}=33, c_{3}=4, c_{4}=16$ e $c_{5}=4$.

$\xi_{1}=89,17 ; \quad \xi_{2}=310 ; \quad \xi_{3}=35,01 ; \quad \xi_{4}=51,88$ e $\eta=4,53 ; W\left(t_{0}\right)=962 e$ $W(T)=1775,9$.

resultando:

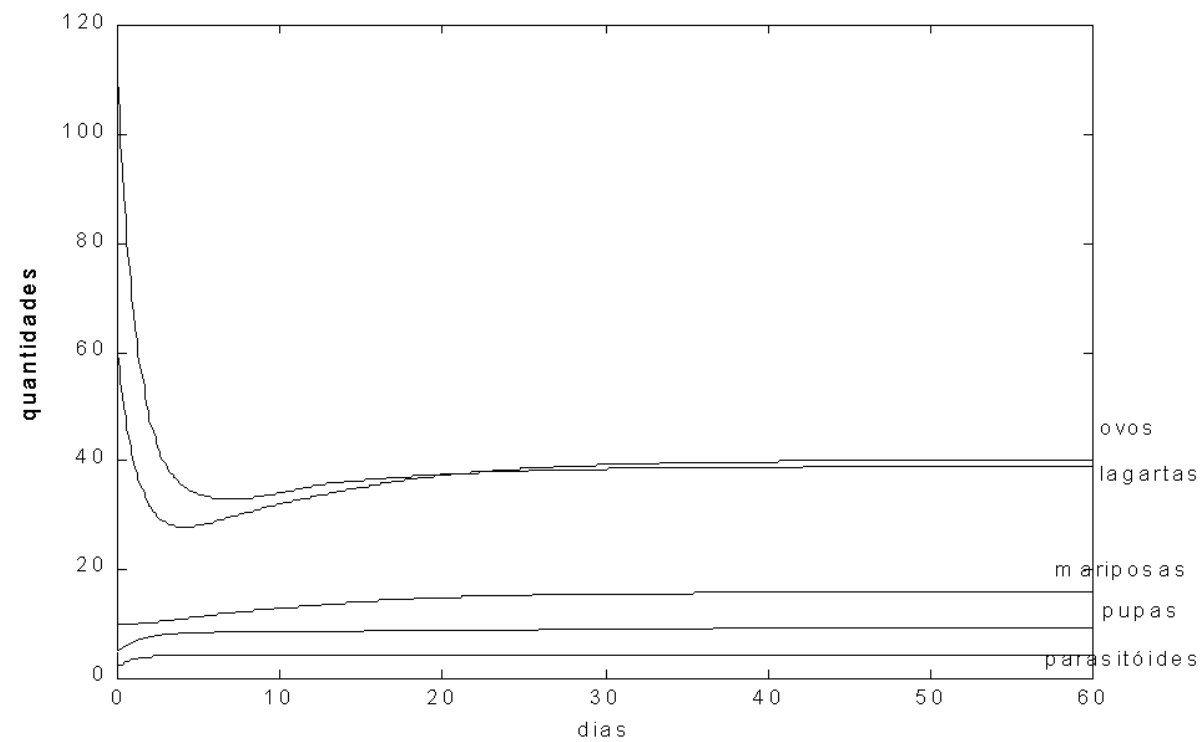

Figura 1: Evolução das pragas e parasitóides com a aplicação de controle

Conclusões:

não é necessário retirar mariposas, pois $B=10 \leq \xi_{1}=89,17$;

não é necessário retirar ovos, pois $O=60 \leq \xi_{2}=310$;

é necessário retirar lagartas, pois $X=110>\xi_{3}=35,01$; 

$\left.m^{2}\right)$

(a quantidade a ser retirada corresponde a $X-\xi_{3}=110-35=75$ lagartas por

não é necessário retirar pupas, pois $P=5 \leq \xi_{4}=51,88$;

é necessário introduzir parasitóides, pois $Y=2<\eta=4,53$;

(a quantidade a ser introduzida corresponde a $\eta-Y=4,53-2 \cong 3$ parasitóides por $\left.m^{2}\right)$.

$2^{a}$ SIMULAÇÃO: sem aplicação de controle

O sistema (1.1), considerando: $U_{1}=U_{2}=U_{3}=U_{4}=V=0$, ou seja, não utilizando as funções de controle, assume a forma seguinte:

$$
\left\{\begin{array}{l}
\frac{d B}{d t}=k_{p} P-\gamma_{b} B^{2}-\mu_{b} B \\
\frac{d O}{d t}=q B-\gamma_{o} O^{2}-\mu_{o} O-k_{o} O \\
\frac{d X}{d t}=k_{o} O-\gamma_{x} X^{2}-\mu_{x} X-\alpha X Y-k_{x} X \\
\frac{d P}{d t}=k_{x} X-\gamma_{p} P^{2}-\mu_{p} P-k_{p} P \\
\frac{d Y}{d t}=\beta X Y-\mu_{y} Y
\end{array}\right.
$$

Mantendo-se os mesmos parâmetros e coeficientes utilizados na simulação anterior, encontra-se $W\left(t_{0}\right)=962$ e $W(T)=1982,4$.

Resultando:

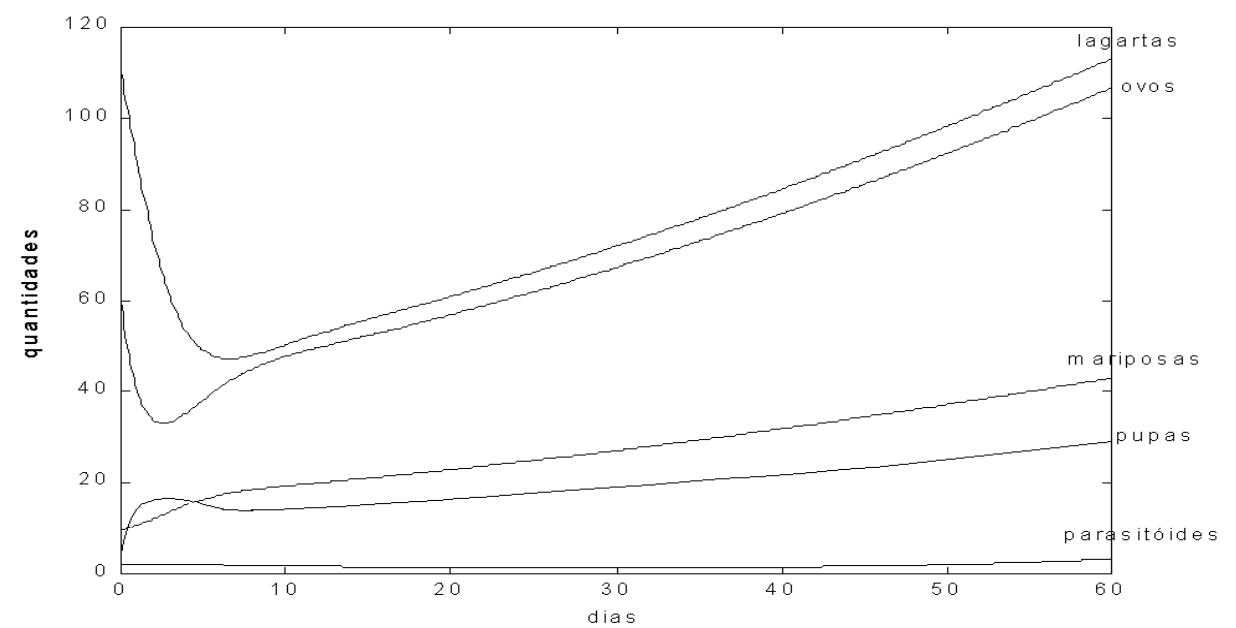

Figura 2: Evolução das pragas e parasitóides sem a aplicação de controle 


\title{
5. Conclusões
}

A solução do sistema (1.1) utilizando o Princípio do Máximo de Pontryagin busca encontrar o nível de pragas abaixo do nível de danos econômicos e um baixo custo no uso de aplicações de controle. Esta solução consiste portanto na minimização dos valores das funções de controle e das populações de pragas durante o período da aplicação e na maximização da população de inimigos naturais. A obtenção desses resultados corresponde a minimizar o funcional (1.4) satisfazendo o sistema (1.1) e as condições (1.2) e (1.3).

$\mathrm{Na} 1^{a}$ simulação, com a aplicação das funções de controle, buscou-se o melhor resultado prático. O nível das lagartas mantém-se estabilizado no patamar considerado como aceitável pela EMBRAPA. Para tanto é necessária a retirada parcial de lagartas e a introdução de parasitóides.

$\mathrm{Na} 2^{a}$ simulação, devido a inexistência das funções de controle, verifica-se que a quantidade de pragas é sempre crescente mantendo-se acima do nível considerado como aceitável pela EMBRAPA, o que reforça a validada da aplicação do controle

\begin{abstract}
One of the most important conquests in pest control research in the last few years was the creation and implementation of the technology of applying Baculovirus in soybean plantations. It was shown that the Baculovirus efficiently combats Anticarsia gemmatalis, a soybean caterpillar. However, one of the main restrictions in the use of Baculovirus is the climate. The application of Baculovirus is more efficient with a humid weather; in such case the disease spreads over the plantation and stays there for a long time. When the climate is dry, as it happened in Rio Grande do Sul on the 1999/2000 crop, the efficiency of Baculovirus and other soybean pest diseases are very decreased. There is also a permanent threat of a selection of individuals more resistant to diseases, due to the application of Baculovirus and other pathogens in successive pest generations. Therefore, several researchers started to search other manners of biological pest control. A well succeeded, in some cases, kind of biological control is the release of parasitoids.

It was considered, in this paper, a mathematical model made of five differential equations, which describes the stages of pest development, and the existent dynamic interactions between them and their parasitoids. It was formulated a control application optimization problem, which minimizes the population of pests and the costs of application and maximizes the population of natural enemies. The problem was solved by the Pontryagin Maximal Principle [1].
\end{abstract}

\section{Referências}

[1] L.S. Pontryagin, V.G. Boltyanskii, R.V. Gamkrelidze e E.F. Mischenko, The mathematical theory of optimal processes, Interscience Publishers, Inc., New York, 1962. 